\title{
Differential epigenetic modifications in the FMR1 gene of the fragile $X$ syndrome after reactivating pharmacological treatments
}

\author{
Elisabetta Tabolacci ${ }^{1,4}$, Roberta Pietrobono ${ }^{1,4}$, Umberto Moscato $^{2}$, Ben A Oostra ${ }^{3}$, Pietro \\ Chiurazzi $^{1}$ and Giovanni Neri*,1 \\ ${ }^{1}$ Istituto di Genetica Medica, Università Cattolica, Rome, Italy; ${ }^{2}$ Istituto di Igiene, Università Cattolica, Rome, Italy; \\ ${ }^{3}$ Department of Clinical Genetics, Erasmus Medical Center, Erasmus University, Rotterdam, The Netherlands
}

The fragile $X$ syndrome is caused by a $>200$ CGG repeat expansion within the FMR 1 gene promoter, with consequent DNA hypermethylation and inactivation of its expression. To further clarify the mechanisms that suppress the activity of the mutant gene and the conditions that may permit its reactivation, we investigated the acetylation and methylation status of three different regions of the FMR1 gene (promoter, exon 1 and exon 16) of three fragile $X$ cell lines, using a chromatin immunoprecipitation (ChIP) assay with antibodies against acetylated-H3/ $\mathrm{H} 4$ histones and against dimethylated lysine residues $\mathrm{K} 4$ and $\mathrm{K} 9$ of histone H3 (H3-K4 and H3-K9). We then coupled the ChIP assay with real-time PCR, obtaining absolute quantification of immunoprecipitated chromatin. Basal levels of histone acetylation and H3-K4 methylation were much higher in transcriptionally active wild-type controls than in inactive fragile $X$ cell lines. Treatment of fragile $X$ cell lines with the DNA demethylating drug 5-aza-2-deoxycytidine (5-azadC), known to reactivate the FMR1 gene, induced a decrease of $\mathrm{H} 3-\mathrm{K} 9$ methylation, an increase of $\mathrm{H} 3$ and $\mathrm{H} 4$ acetylation and an increase of H3-K4 methylation. Treatment with acetyl-L-carnitine (ALC), a compound that reduces the in vitro expression of the FRAXA fragile site without affecting DNA methylation, caused an increase of $\mathrm{H} 3$ and $\mathrm{H} 4$ acetylation. However, $\mathrm{H} 3-\mathrm{K} 4$ methylation remained extremely low, in accordance with the observation that ALC alone does not reactivate the FMR1 gene. Our experiments indicate that H3-K4 methylation and DNA demethylation are the main epigenetic switches activating the expression of the FMR1 gene, with histone acetylation playing an ancillary role.

European Journal of Human Genetics (2005) 13, 641-648. doi:10.1038/sj.ejhg.5201393

Published online 2 March 2005

Keywords: fragile $X$ syndrome; reactivating treatments; epigenetic modifications; chromatin immunoprecipitation; real-time PCR

\footnotetext{
*Correspondence: Professor G Neri, Istituto di Genetica Medica, Università Cattolica, Largo F. Vito 1, 00168 Rome, Italy. Tel: + 3906305 4449; Fax: + 39063050031 ;

E-mail: gneri@rm.unicatt.it

${ }^{4}$ These two authors contributed equally to this work.

Received 8 September 2004; revised 18 November 2004; accepted 25 January 2005
}

Introduction
The fragile X syndrome (OMIM +309550 ) is the most
common inherited form of mental retardation. It is caused
by the expansion of a polymorphic CGG trinucleotide
repeat in the promoter of the FMR1 gene, consisting of
more than 200 repeats (full mutation) instead of $6-54$
CGG repeats, as in normal alleles. ${ }^{1,2}$ In affected patients,
cytosines of the CGG expansion and of the upstream
CpG island are abnormally methylated and lead to 
transcriptional inactivation of the gene ${ }^{3,4}$ and absence of the FMR1 protein (FMRP). FMRP is an mRNA-binding protein, whose absence was recently shown to cause a translational dysregulation of several mRNAs normally associated with FMRP, allowing the identification of candidate genes relevant to this phenotype., ${ }^{5,6}$ Rare individuals of normal intelligence have been described, who express FMRP and harbor a completely or partially unmethylated full mutation, clearly indicating that DNA methylation is the key factor in silencing FMR1. ${ }^{7,8}$

We previously demonstrated that treatment of fragile $\mathrm{X}$ lymphoblastoid cell lines with the DNA methylation inhibitor 5-aza-2-deoxycytidine (5-azadC) leads to the transcriptional reactivation of the mutant FMR1 gene. ${ }^{9}$ We then studied single-cell methylation by analyzing the promoter region of the gene with the bisulfite-sequencing technique and found that a large proportion of cells (70$90 \%$ ) became unmethylated after 5 -azadC treatment. ${ }^{10}$ On the other hand, the amount of FMR1 mRNA, measured by quantitative fluorescent RT-PCR, indicated that transcriptional reactivation efficiency was only 15-20\% in comparison to a normal cell line, ${ }^{10}$ possibly due to a residual methylation of the CGG repeat. Our results confirmed the role of DNA methylation in the transcriptional silencing of the FMR1 gene.

Research over the past few years focused on the direct and indirect mechanisms by which DNA methylation mediates gene silencing. DNA methylation can directly inhibit binding of transcription factors to gene promoters, while indirect mechanisms are mediated by local chromatin changes. Methylated $\mathrm{CpG}$ residues can then be recognized by methyl binding proteins, such as MeCP2 and MBD1-4, which recruit histone deacetylases (HDACs). ${ }^{11,12}$ HDACs remove acetyl groups from lysines of histone $\mathrm{H} 3$ and $\mathrm{H} 4$ amino-terminal tails, eventually leading to a more condensed chromatin structure (heterochromatin). ${ }^{13}$ In normal controls, the FMR1 gene is associated with acetylated histones $\mathrm{H} 3$ and $\mathrm{H} 4$, while acetylation is reduced in fragile $\mathrm{X}$ cells. ${ }^{14}$ Increased acetylation of FMR1-associated histone $\mathrm{H} 3$ and $\mathrm{H} 4$ was induced by treatment with 5-azadC and with the HDAC inhibitor trichostatin A. ${ }^{14}$ We then reported that histone hyperacetylating drugs (HDAC inhibitors), such as sodium butyrate (BA) and 4-phenylbutyrate (4-PBA), synergistically potentiate the FMR1 gene reactivation induced by 5azadC. ${ }^{15}$ This observation confirms that CpG cytosine methylation and histone deacetylation cooperate in silencing chromatin domains.

Histone amino-terminal tails can be modified through the attachment (or removal) of acetyl-, phosphate- or methyl-groups. ${ }^{16}$ Recently, attention has been focused on the methylation of histone $\mathrm{H} 3$ at lysines 4 and 9 (H3-K4 and H3-K9), which play an important role in transcriptional regulation. In particular, H3-K4 methylation correlates with activation of gene expression (high in euchromatin) and H3-K9 methylation with transcriptional inactivation (high in heterochromatin). ${ }^{17,18}$ Coffee et al ${ }^{19}$ reported that exon 2 of the FMR1 gene has decreased H3-K4 and increased H3-K9 methylation, in accordance with its silent status, and that 5 -azadC treatment induces an increase of H3-K4 methylation, but only a transient decrease of H3-K9 methylation.

Here we present a detailed analysis of the epigenetic modifications of the FMR1 gene in normal and fragile $\mathrm{X}$ cell lines, using a chromatin immunoprecipitation (ChIP) protocol coupled with real-time PCR, which allows better quantitation of epigenetically modified histones. In contrast to previous reports, ${ }^{14,19}$ we tested three different regions of the FMR1 gene (promoter region, exon 1 next to the CGG repeat tract and exon 16) and found significant differences before and after 5-azadC treatment. All ChIP experiments were accompanied by measurement of FMR1 mRNA levels by quantitative RT-PCR, so that a correlation between epigenetic modifications and transcriptional activity of the gene could be established. We also report on the epigenetic effect of acetyl-L-carnitine (ALC), a drug which reduces the cytogenetic expression of the fragile site FRAXA ${ }^{20}$ possibly through direct histone acetylation.

\section{Materials and methods Cell cultures and drugs treatments}

Lymphoblastoid cell lines were established by Epstein-Barr virus (EBV) transformation from peripheral blood lymphocytes of male fragile $X$ patients and two normal control males. The three fragile $X$ cell lines used in these experiments were: E3 carrying an FMR1 allele with 250 CGG repeats, S1 with 450 CGGs and E6 with over 550 CGG repeats. Cells were grown in RPMI1640 medium with $10 \%$ fetal calf serum and penicillin/streptomycin at $37^{\circ} \mathrm{C}$ with $5 \% \mathrm{CO}_{2}$. Medium was changed every $48 \mathrm{~h}$. Cells were treated daily with $1 \mu \mathrm{M}$ 5-azadC (Sigma) for 7 days. Treatment with $10 \mathrm{~mm}$ ALC (Sigma-Tau) was also for 7 days, with only two additions of the drug.

\section{RNA extraction and reverse transcriptase-PCR}

Total RNA from treated and untreated cell lines was extracted with the single-step acid phenol method, using Trizol (Invitrogen). RNA was reverse-transcribed by MoMLV-reverse transcriptase (Gibco-BRL). PCR was performed as previously described, ${ }^{9}$ employing $1 \mu \mathrm{l}$ of cDNA with specific primers for FMR1 and for the housekeeping gene hypoxanthine guanine phosphorybosyltransferase (HPRT) as internal control. PCR conditions were as follows: initial denaturation at $95^{\circ} \mathrm{C}$ for $5 \mathrm{~min}$, followed by 35 cycles at $95^{\circ} \mathrm{C}$ for $1 \mathrm{~min}$, at $55^{\circ} \mathrm{C}$ for $1 \mathrm{~min}$ and at $72^{\circ} \mathrm{C}$ for $2 \mathrm{~min}$ and then a final step of extension at $72^{\circ} \mathrm{C}$ for $5 \mathrm{~min}$. 


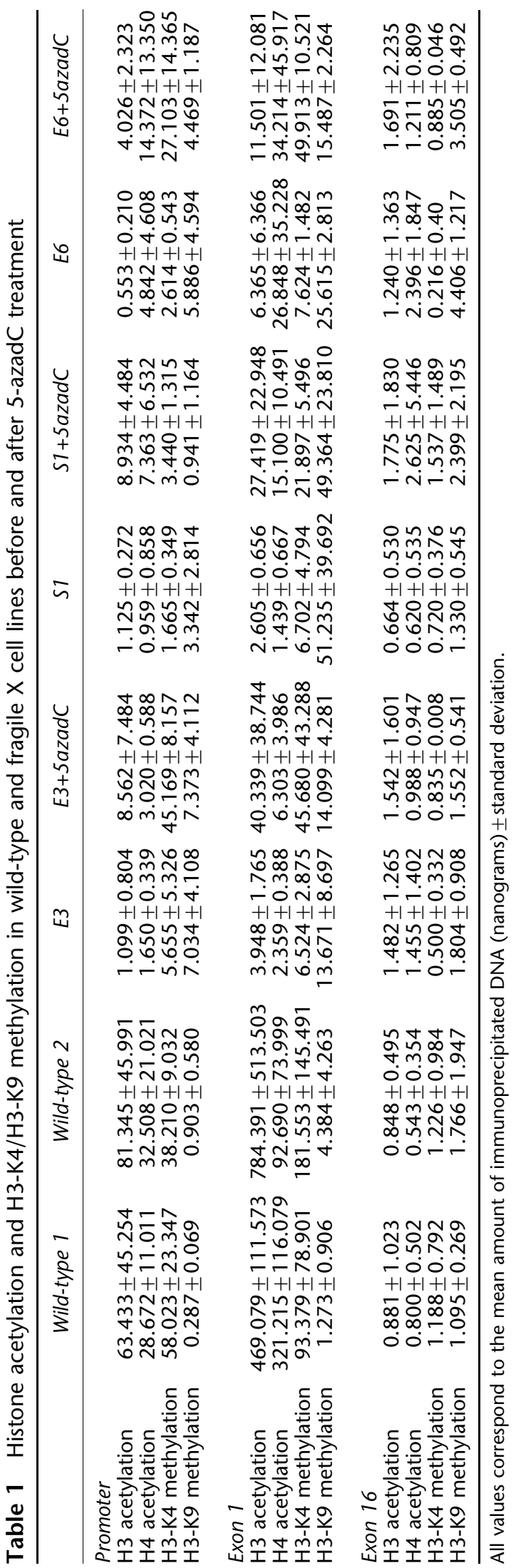

\section{Quantitative RT-PCR analysis}

For a quantitative estimate of the relative FMR1 mRNA levels, we adapted the technique described by Tassone et $a^{21}{ }^{21}$ using an ABI 7700 Sequence Detector with duallabeled TaqMan probes. The FMR1 amplicon is a 89-bp product spanning the junction between exon 13 and 14 of the gene (positions 1432-1520 of GenBank sequence NM_002024). The following primers and TaqMan probe were employed: forward 5'-GGA ACA AAG GAC AGC ATC GC-3', reverse $5^{\prime}$-CTC TCC AAA CGC AAC TGG TCT-3'; TaqMan probe $5^{\prime}$-(FAM)-AAT GCC ACT GTT CTT TTG GAT TAT CAC CTG AA-(TAMRA)-3'. The relative amount of FMR1 mRNA was assessed by comparison with the human HPRT mRNA detected with the Pre-Developed TaqMan Assay Reagent ABI 4310890E (huHPRT endogenous control). Final reaction volume was $25 \mu \mathrm{l}$ in the TaqMan Universal PCR Master Mix (ABI 4304437) with $900 \mathrm{nM}$ of each primer and $100 \mathrm{~nm}$ dual-labeled probe for FMR1. Cycle parameters were $2 \mathrm{~min}$ at $50^{\circ} \mathrm{C}$ and $10 \mathrm{~min}$ at $95^{\circ} \mathrm{C}$, followed by 40 cycles of $15 \mathrm{~s}$ at $95^{\circ} \mathrm{C}$ denaturation and $1 \mathrm{~min}$ at $60^{\circ} \mathrm{C}$ annealing/extension. Relative FMR1 levels were calculated as follows: $2^{-[\Delta C t(\text { fragile } \mathrm{X})-\Delta C \mathrm{t}(\text { control })]}=$ $2^{-\Delta \Delta C \mathrm{t}}$, where $\Delta C_{\mathrm{t}}$ equals $C_{\mathrm{t}}(F M R 1)-C_{\mathrm{t}}(H P R T)$ as discussed by Tassone et al. ${ }^{21}$

\section{ChIP assay}

We employed the ChIP Assay Kit and antibodies from Upstate Biotechnology and followed the manufacturer's protocol. Histone acetylation was studied by ChIP with antibodies directed against histone $\mathrm{H} 4$ acetylated at lysines 5, 8, 12 and 16, and histone H3 acetylated at lysines 9 and 14 . Histone methylation analysis was performed using two different antibodies against histone H3 dimethylated at lysine 9 or at lysine 4. After ChIP, DNA was extracted with a standard procedure (phenol/chloroform/isoamilic alcool) (25:24:1) and digested with XhoI, prior to quantitative fluorescent PCR analysis, in order to separate the CGG repeats, which could interfere with amplification efficency.

\section{Quantification of immunoprecipitated DNA (IP-DNA) by real-time PCR}

The levels of acetylation of histone $\mathrm{H} 3$ and $\mathrm{H} 4$ and of methylation of lysines H3-K9 and H3-K4 of both FMR1 and HPRT were measured by fluorescent PCR by ABI 7700 Sequence Detector with dual-labeled TaqMan probes.

Primers and probes employed for PCR analysis were designed to amplify three different portions of the FMR1 gene: the promoter region (positions 13376-13448 of GenBank L29074), exon 1 near the CGG repeat tract (positions 13940-14012 of GenBank L29074) and the last amplicon overlapping exon 16 (positions 47305-47386 of GenBank L29074). For HPRT, the amplified amplicon was 
that overlapping exon 6 (positions 34946-35022 of GenBank sequence M26434). The sequences of primers and probes were as follows: 5'-AACTgggATAACCggATgCAT-3' forward, 5' -ggCCAgAACgCCCATTTC- $3^{\prime}$ reverse and 5' (FAM)TgATTTCCCACgCCACTgAgTgCAC (TAMRA)-3' probe for the promoter region; 5'-CgCTAgCAgggCTgAAgAgA-3' forward, 5'-CTTgTAgAAAgCgCCATTggA-3' reverse and 5'(FAM)AtggAggAgCTggTggTggAAgTgC (TAMRA)-3' probe for exon 1; 5'-AATATTCTCATAggAAACgACgATCA-3' forward, 5' CTgTTgTTCTTCCTTTAgCCTCTC TT- $3^{\prime}$ reverse and $5^{\prime}$-(FAM) TCCCgAACAgATAATCgTCCACgTAATCC (TAMRA)-3' probe for exon 16; 5'-TgACACTggCAAAACAATgCA-3' forward, 5'-CCTTgCgACCTTgACC ATCT-3' reverse and 5' -(VIC)TTTgC TTTCCTTggTCAggCAgTATAATCCA(TAMRA)-3' probe for $H P R T$ exon 6. Cycle parameters were $2 \mathrm{~min}$ at $50^{\circ} \mathrm{C}$ and $10 \mathrm{~min}$ at $95^{\circ} \mathrm{C}$, followed by 40 cycles of $15 \mathrm{~s}$ at $95^{\circ} \mathrm{C}$, for denaturation and $1 \mathrm{~min}$ at $60^{\circ} \mathrm{C}$ for annealing/ extension. A standard curve for the three FMR1 and for the single HPRT amplicon was constructed with five different DNA dilutions of known concentration (X-axis $=\log [X])$ and the corresponding $C_{\mathrm{t}}$ values ( $Y$-axis). The unknown amount of acetylated and methylated IP-DNA of FMR1 and HPRT $(X$-axis $=\log [X])$ was calculated from $C_{\mathrm{t}}$ values, through the standard curve plot. Normalized FMR1 levels were estimated dividing the amount of FMR1 IP-DNA by the amount of HPRT. The acetylation and methylation levels of the treated cell lines has been compared to the levels of untreated and/or control male cell lines.

\section{Statistical analysis}

All variables were analyzed by means of descriptive statistics (mean, median, standard deviation and standard error of mean) and the $\tau$-test was used to compare the means of the continuous variables in the untreated and treated group. We tested the null hypotesis that 5-azadC and ALC treatments did not induce any increase of the $\mathrm{H} 3-\mathrm{H} 4$ acetylation or changes in $\mathrm{K} 4$ and $\mathrm{K} 9$ methylation in the three regions of the FMR1 gene analyzed in the three cell lines. The $F$-test was employed to explain the total variation, evaluating the ratio between the values before $($ value $=1$ ) and after treatments. If $\tau$-test or $F$-ratio were near 1 , the null hypothesis was valid; on the contrary, if $\tau$ test or F-ratio were $>1$, the null hypothesis was rejected.

All tests were two-tailed, and the level of significance was 0.05. The collection and analysis of data were performed using the Statistical Package for Social Sciences Release 6.0 (SPSS; Chicago, IL, USA).

\section{Results}

Table 1 summarizes the results of ChIP experiments performed on two wild-type and three fragile $\mathrm{X}$ cell lines before and after 5-azadC treatment, while Figure 1 illustrates in graphical form only the results of one fragile X cell line (E6) as example. The data in Table 1 clearly demonstrate that histone acetylation and H3-K4 methylation levels in the promoter and exon 1 region were significantly higher in wild-type controls, while H3-K9

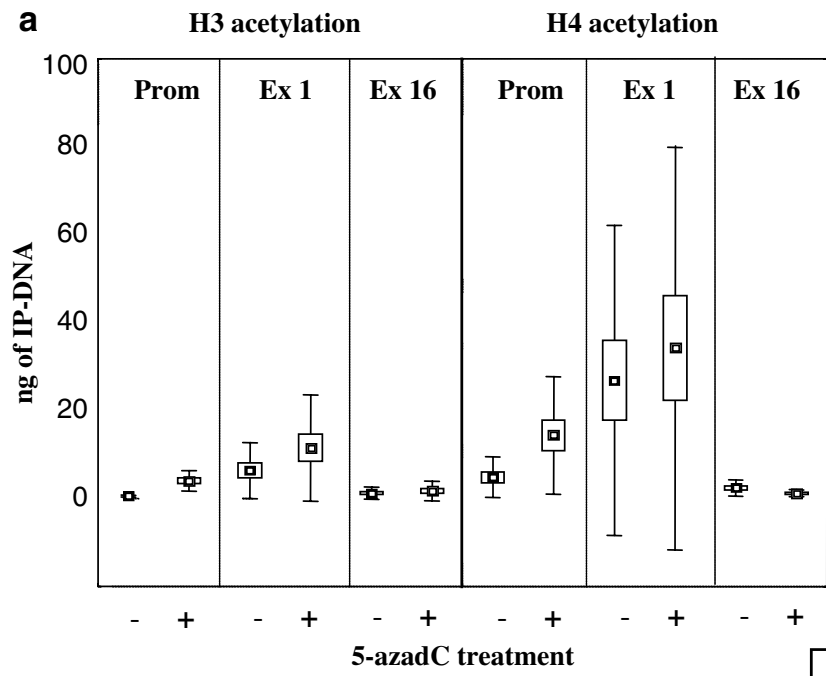

b

b H3-K4 methylation H3-K9 methylation

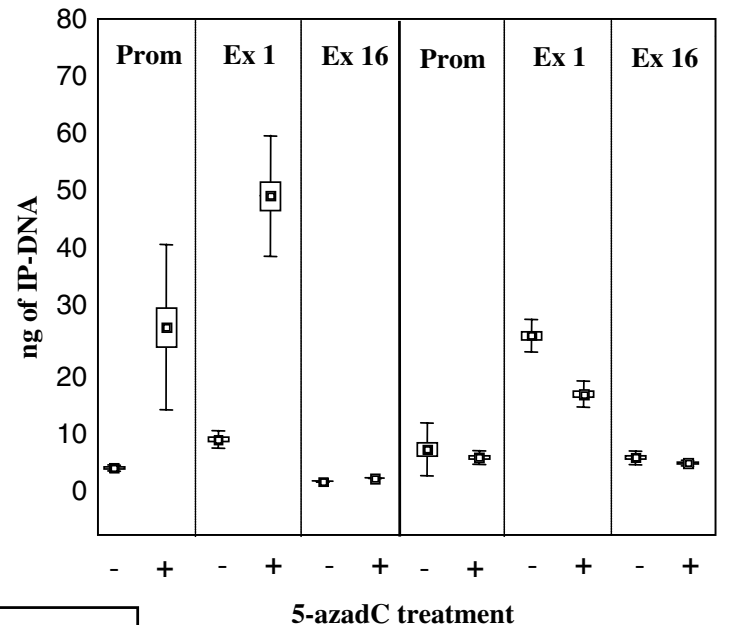

$$
\begin{array}{|l|}
\mp \text { Mean } \pm \text { SD } \\
\square \text { Mean } \pm \text { SE } \\
\square \text { Mean } \\
\hline
\end{array}
$$

Figure 1 ChIP analysis after $1 \mu \mathrm{M}$ 5-azadC treatment for 7 days of histone $\mathrm{H} 3$ and $\mathrm{H} 4$ acetylation (a) and of histone $\mathrm{H} 3-\mathrm{K} 4$ and $\mathrm{H} 3-\mathrm{K} 9$ methylation (b) in the E6 fragile X cell line. Each box-plot depicts the amount (ng) of IP-DNA in three different regions of the FMR1 gene, corresponding to the promoter, exon 1 and exon 16. The plus and minus signs beneath each box-plot indicate whether treatment had been administered ( + ) or not $(-)$. 


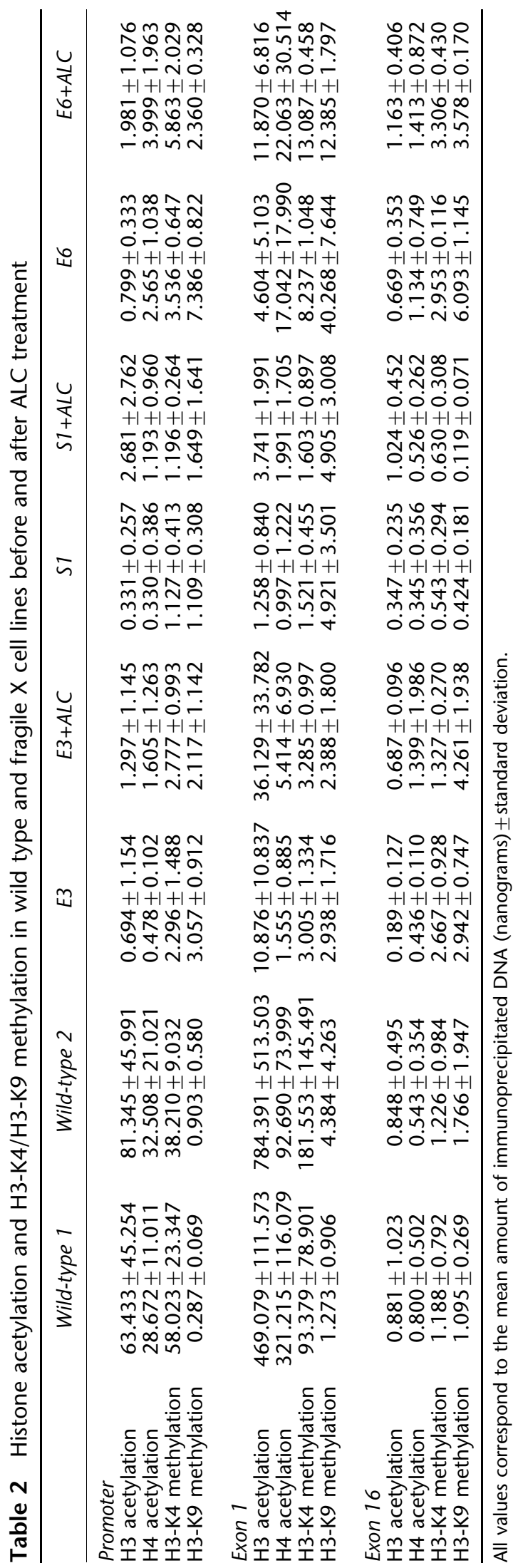

methylation was significantly higher in fragile $\mathrm{X}$ cell lines. Treatment with 5 -azadC of the three fragile $X$ cell lines induced an increase in histone acetylation. This increase was limited to the promoter and exon 1 regions, while no significant difference was observed in the region corresponding to exon 16 (Figure 1a). Likewise, H3-K4 methylation increased significantly in all three fragile $\mathrm{X}$ cell lines, again more pronouncedly around the promoter and exon 1 region, but not in exon 16 (Figure $1 \mathrm{~b}$ ). These epigenetic changes correlated with the transcriptional reactivation of the gene, which amounted to $8-15 \%$ of wild-type values, as measured by quantitative fluorescent RT-PCR, in all three fragile $X$ cell lines (data not shown). In accordance with previous results, ${ }^{19}$ we observed a significant reduction in H3-K9 methylation levels in fragile $X$ cell line $\mathrm{E} 6$ (Figure 1b). However, H3-K9 methylation did not change after 5-azadC treatment in fragile $\mathrm{X}$ cell lines $\mathrm{S} 1$ and $\mathrm{E} 3$ (Table 1).

The increased levels of $\mathrm{H} 3 / \mathrm{H} 4$ acetylation after 5-azadC treatment, though significant, did not reach the corresponding levels of wild-type controls. In fact, histone acetylation in the promoter and exon 1 region of fragile $\mathrm{X}$ cell lines rose at most to $10 \%(\mathrm{H} 3)$ and $30 \%(\mathrm{H} 4)$ of wildtype levels after 5-azadC treatment (Table 1). Histone acetylation in exon 16 was extremely low in both wild-type and fragile $\mathrm{X}$ cell lines and was not affected by treatment. The 5-azadC treatment also induced a significant increase of H3-K4 methylation, approximately $10-50 \%$ of levels found in wild-type controls, for the promoter and exon 1 region, respectively. Once again, H3-K4 methylation in exon 16 was low and unchanged by treatment (Table 1 ).

ChIP results obtained before and after treatment with ALC are summarized in Table 2, while the experiments performed on the E6 cell line are illustrated in Figure 2. All three fragile $X$ cell lines showed an increase, although modest, of $\mathrm{H} 3$ and $\mathrm{H} 4$ acetylation levels in the promoter and exon 1 region. Treatment with ALC did not modify histone acetylation status in exon 16, similar to what was observed after 5-azadC treatment (Figure 2a). As with 5azadC treatment, the increased levels of histone acetylation induced by ALC remained lower than those of wildtype controls. However, in contrast to 5-azadC, treatment with ALC (10 mM for 7 days) did not induce reactivation of the FMR1 gene, as mRNA levels were measured by quantitative fluorescence RT-PCR and remained undetectable in all three fragile X cell lines (data not shown). Failure of FMR1 reactivation correlates with the extremely low levels of H3-K4 methylation, which did not increase at all after ALC treatment in any of the fragile $X$ cell lines (Figure 2b). Interestingly, levels of H3-K9 methylation in the exon 1 region did decrease in E6 (Figure 2b) but not in the other two fragile $X$ cell lines (E3 and S1). Table 3 summarizes the epigenetic changes that we observed in the promoter and exon 1 region of the FMR1 gene in the tested cell lines before and after treatments. 


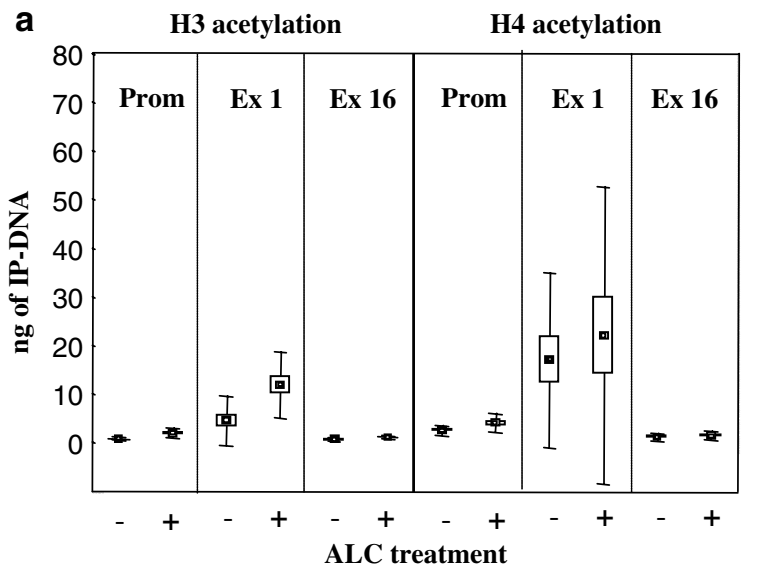

b H3-K4 methylation H3-K9 methylation

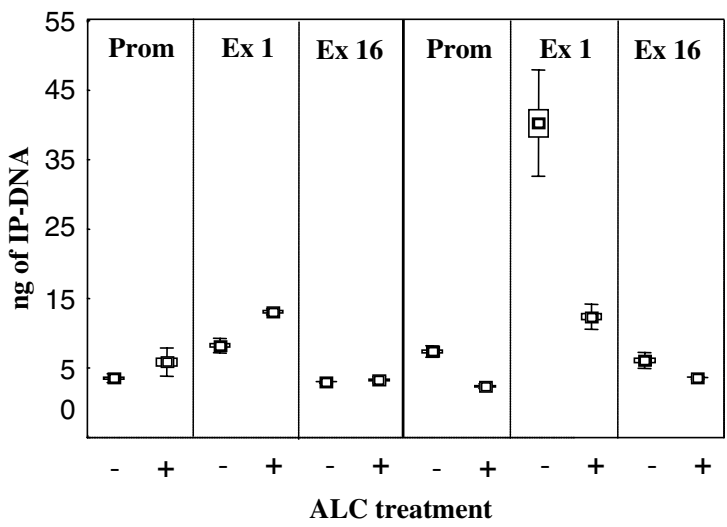

$$
\begin{array}{|l}
\hline \text { Mean } \pm \text { SD } \\
\square \text { Mean } \pm \text { SE } \\
\square \text { Mean }
\end{array}
$$

Figure 2 ChIP analysis after $10 \mathrm{~mm} \mathrm{ALC}$ treatment for 7 days of histone $\mathrm{H} 3$ and $\mathrm{H} 4$ acetylation (a) and of histone $\mathrm{H} 3-\mathrm{K} 4$ and $\mathrm{H} 3-\mathrm{K} 9$ methylation (b) in the E6 fragile X cell line. Each box-plot depicts the amount (ng) of IP-DNA in three different regions of the FMR1 gene, corresponding to the promoter, exon 1 and exon 16. The plus and minus signs beneath each box-plot indicate whether treatment had been administered $(+)$ or not $(-)$.

Table 3 Summary of the observed epigenetic changes in a methylated full mutation before and after 5-azadC and ALC treatment, compared to a wild-type cell line

\begin{tabular}{lcccc}
\hline & Wild-type & Full & Full+5azadC & Full+ALC \\
\hline DNA methylation & - & + & - & $+^{\mathrm{a}}$ \\
Histone H3 acetylation & + & - & + & + \\
Histone H4 acetylation & + & - & + & + \\
H3-K4 methylation & + & - & + & $+/-$ \\
H3-K9 methylation & - & + & $+/-$ & $+/-$ \\
FMR1 mRNA & + & - & + & - \\
\hline
\end{tabular}

${ }^{\mathrm{a}}$ Data reported by Pascale et al. ${ }^{24}$

\section{Discussion}

We have previously shown that transcription and translation of a methylated full mutation can be partially restored by treating fragile $\mathrm{X}$ cells with the DNA demethylating agent 5 -azadC. ${ }^{9,10}$ We also showed that compounds known to increase histone acetylation can act synergistically with 5 -azadC. ${ }^{15}$ To better understand the mechanisms of the FMR1 gene reactivation, we undertook a systematic study of its epigenetic status. In particular, we tested acetylation and methylation of histones $\mathrm{H} 3$ and $\mathrm{H} 4$, in three different regions of the gene, that is, promoter, exon 1 and exon 16 . As expected, we found that the levels of histone $\mathrm{H} 3$ and $\mathrm{H} 4$ acetylation are much higher in wild-type (FMR1-active) cells, as compared to fragile X (FMR1-inactive) cells. Similar results had been obtained by Coffee et al, ${ }^{14}$ although these authors only investigated the epigenetic status of FMR1 intron 1 , approximately $200 \mathrm{bp}$ downstream of exon 1 region. By analyzing multiple regions of the gene, we found that transcriptional reactivation of three fragile $\mathrm{X}$ cell lines with 5-azadC was paralleled by increased acetylation of both $\mathrm{H} 3$ and $\mathrm{H} 4$, exclusively in the promoter region and in exon 1 . The exon 16 region remained substantially unmodified, possibly because its acetylation level is very low already before treatment, as in wild-type cells, indicating that the epigenetic modifications relevant to the transcription of the FMR1 gene are concentrated in the $5^{\prime}$ region. We did not observe a clear correlation between the extent of the tested epigenetic modifications and the size of the CGG expansion, among the three fragile $\mathrm{X}$ cell lines after 5-azadC treatment. In future studies, it might be interesting to test other tracts of the gene in order to characterize the extent of the active chromatin domain in the normal and reactivated FMR1 gene.

Methylation of H3-K4, normally much lower in fragile $\mathrm{X}$ cells compared to wild type, increased consistently after 5 -azadC treatment, in the promoter and exon 1 region. Again, the relationship with the levels of transcriptional reactivation was not linear, given that the maximum reactivation was observed in line $\mathrm{E} 3$, rather than in line E6, which had higher H3-K4 methylation levels.

H3-K9 residue was definitely more methylated in exon 1 of fragile X cell lines when compared to wild-type controls, representing a localized epigenetic mark, characteristic for heterochromatin. ${ }^{22}$ However, no significant reduction of H3-K9 methylation was induced by 5-azadC in the E3 and S1 fragile X cell line. However, a 40\% decrease was observed in exon 1 of line E6. Our results suggest that increases in $\mathrm{H} 3$ and $\mathrm{H} 4$ acetylation and in $\mathrm{H} 3-\mathrm{K} 4$ 
methylation, in addition to DNA demethylation, are critical for the transition from a transcriptionally nonpermissive to a transcriptionally permissive configuration of the FMR1 gene chromatin, as depicted schematically in Figure 3 . The model is only an approximation and does not explain why the transcriptional reactivation is never complete. This could be due to incomplete demethylation of the CGG tract, ${ }^{3}$ or else to the fact that only a proportion of cells respond to the reactivating treatment, as previously discussed. ${ }^{10}$ On the other hand, variations in the level of H3-K9 methylation seem to be less critical with respect to the transition from heterochromatin to euchromatin.

To further dissect the role of DNA and histone methylation from that of histone acetylation, we studied the effect of ALC, a compound that should increase histone acetylation. We had previously shown that ALC inhibits the cytogenetic expression of the fragile site FRAXA. ${ }^{20}$ It also proved effective in ameliorating the hyperactive behavior, when administered to children with the fragile $\mathrm{X}$ syndrome. ${ }^{23}$ However, Pascale et $a l^{24}$ showed that long-term ALC treatment did not cause DNA demethylation of the mutant FMR1 promoter, nor reactivation of the gene. We now show that treatment with ALC of three fragile $\mathrm{X}$ cell lines results in significant acetylation of FMR1-associated $\mathrm{H} 3$ and $\mathrm{H} 4$ histones, especially in the promoter and exon 1 region. Interestingly, H3-K9 methylation was also reduced by ALC in the promoter and exon 1 region of the E6 cell line, a finding correlating with the increased acetylation of histone H3. In fact, H3-K9 can undergo only one of two possible epigenetic modifications, either acetylation or methylation. However, all these changes were not sufficient to restore transcription and, in accordance with the lack of FMR1 reactivation, ALC treatment did not induce a significant increase in the level of H3-K4 methylation.
These results confirm our previous assumption ${ }^{15}$ that DNA methylation, which persists after ALC treatment, ${ }^{24}$ rather than histone acetylation, is the dominant epigenetic factor in the regulation of transcription of the FMR1 gene.

In conclusion, it appears that the epigenetic status of a methylated full mutation tends to be changed into that of a wild-type by treatment with 5 -azadC, while treatment with ALC, which does not cause FMR1 reactivation, affects significantly histone acetylation, but not histone and DNA methylation. Only H3-K4 methylation, particularly around the promoter and exon 1 region, correlates with DNA hypomethylation and gene transcription. In fact, FMR1 is actively transcribed in the full mutation cell lines treated with 5-azadC, that have a methylated H3-K4.

It has been suggested that methylated H3-K9 recruits histone methyltransferases (HMTs) associated with HDACs via the heterochromatic protein $1-\mathrm{HP} 1,{ }^{25,26}$ resulting in histone deacetylation. However, our experiments indicate that H3-K9 methylation and partial histone deacetylation can coexist with H3-K4 methylation and active FMR1 transcription. We reached the same conclusion also by characterizing an exceptional cell line that harbors an unmethylated full mutation. This cell line is transcriptionally active and has high H3-K4 methylation levels, in spite of low histone acetylation and high H3-K9 methylation. ${ }^{27}$

Understanding the molecular mechanisms by which the FMR1 gene is transcriptionally regulated will have significant bearing on the ultimate goal of finding an effective cure for the fragile $\mathrm{X}$ syndrome.

\section{Acknowledgements}

We gratefully acknowledge Steve Warren for providing the fragile $X$ lymphoblastoid cell line S1. This work was supported by the FRAXA

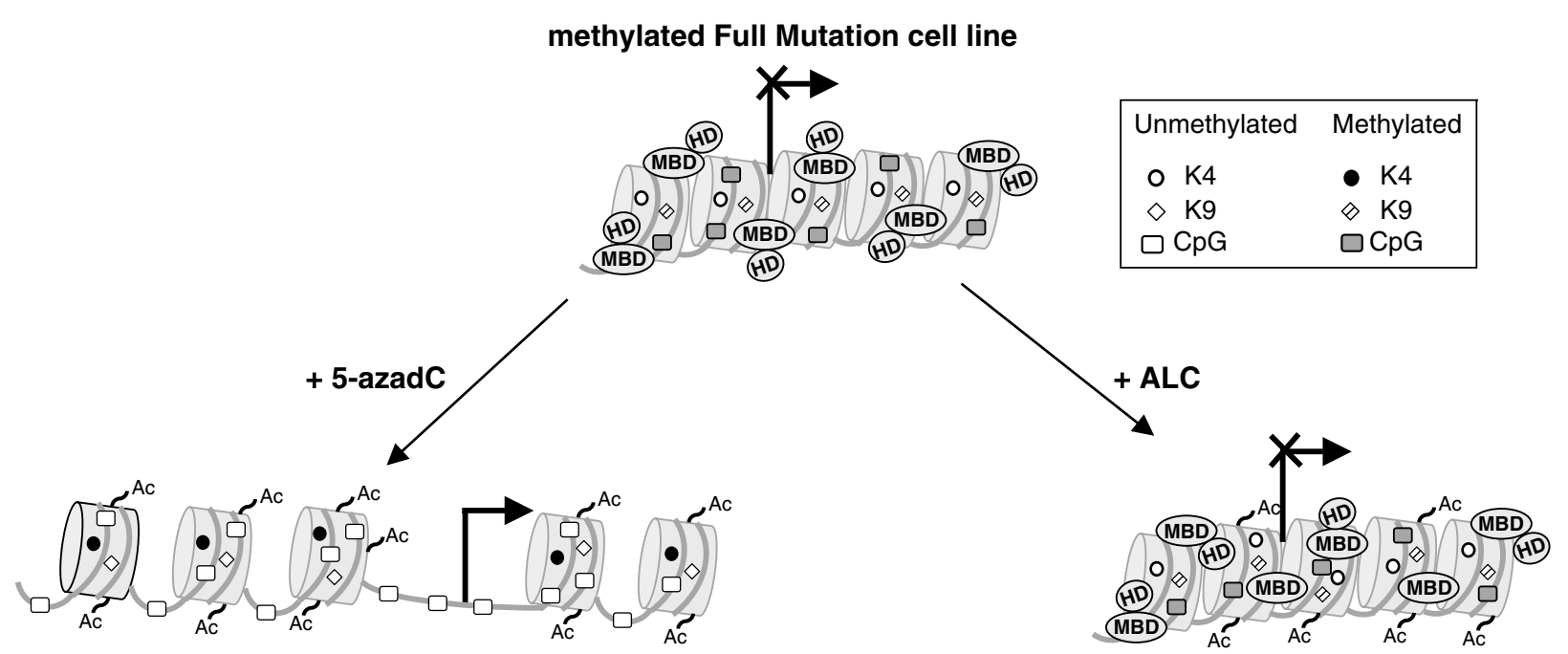

Figure 3 A simplified model of chromatin remodeling after 5-azadC and ALC treatment of a methylated full mutation. MBD: methyl-DNA binding proteins; HD: histone deacetylases; Ac: acetyl groups on histone $\mathrm{H} 3$ and $\mathrm{H} 4$ tails. 
Foundation, Conquer Fragile X, Sigma-Tau, Fondazione Telethon, MIUR, Fondazione Cariplo and Associazione Anni Verdi.

\section{References}

$1 \mathrm{Fu}$ YH, Kuhl DP, Pizzuti A et al: Variation of the CGG repeat at the fragile $\mathrm{X}$ site results in genetic instability: resolution of the Sherman paradox. Cell 1991; 67: 1047-1058.

2 Verkerk AJMH, Pieretti M, Sutcliffe JS et al: Identification of a gene (FMR-1) containing a CGG repeat coincident with a breakpoint cluster region exhibiting lenght variation in fragile $\mathrm{X}$ syndrome. Cell 1991; 65: 905-914.

3 Feng Y, Zhang F, Lokey LK et al: Translational suppression by trinucleotide repeat expansion at FMR1. Science 1995; 268: $731-734$.

4 Pieretti M, Zhang F, Fu YH et al: Absence of expression of the FMR-1 gene in fragile X syndrome. Cell 1991; 66: 817-822.

5 Brown V, Jin P, Ceman S et al: Microarray identification of FMRP-associated brain mRNAs and altered mRNA translational profiles in fragile X syndrome. Cell 2001; 107: 477-487.

6 Zalfa F, Bagni C: Molecular insights into mental retardation: multiple functions for the fragile $\mathrm{X}$ mental retardation protein? Curr Issues Mol Biol 2004; 6: 73-88.

7 Hagerman RJ, Hull CE, Safanda JF et al: High functioning fragile X males: demonstration of an unmethylated fully expanded FMR1 mutation associated with protein expression. Am J Med Genet 1994; 51: 298-308.

8 Smeets HJ, Smits AP, Verheij CE et al: Normal phenotype in two brothers with a full FMR1 mutation. Hum Mol Genet 1995; 4: 2103-2108.

9 Chiurazzi P, Pomponi MG, Willemsen $\mathrm{R}$ et al: In vitro reactivation of the FMR1 gene involved in fragile X syndrome. Hum Mol Genet 1998; 7: 109-113.

10 Pietrobono R, Pomponi MG, Tabolacci E et al: Quantitative analysis of DNA demethylation and transcriptional reactivation of the FMR1 gene in fragile $X$ cells treated with 5-azadeoxycytidine. Nucleic Acids Res 2002; 30: 3278-3285.

11 Jones PL, Veenstra GJC, Wade PA et al: Methylated DNA and $\mathrm{MeCP} 2$ recruit histone deacetylase to repress transcription. Nat Genet 1998; 19: 187-191.

12 Nan X, Ng H-H, Johnson CA et al: Transcriptional repression by the methyl-CpG-binding protein $\mathrm{MeCP} 2$ involves a histone deacetylase complex. Nature 1998; 393: 386-389.
13 Razin A: CpG methylation, chromatin structure and gene silencing - a three-way connection. EMBO J 1998; 17: 49054908.

14 Coffee B, Zhang F, Warren ST et al: Acetylated histones are associated with FMR1 in normal but not fragile X-syndrome cells. Nat Genet 1999; 22: 98-101.

15 Chiurazzi P, Pomponi MG, Pietrobono R et al: Synergistic effect of histone hyperacetylation and DNA demethylation in the reactivation of the FMR1 gene. Hum Mol Genet 1999; 8: $2317-2323$.

16 Turner BM: Cellular memory and the histone code. Cell 2002; 111: $285-291$.

17 Grewal SIS, Moazed D: Heterochromatin and epigenetic control of gene expression. Science 2003; 301: 798-802.

18 Lachner M, O'Sullivan RJ, Jenuwein T: An epigenetic road map for histone lysine methylation. J Cell Sci 2003; 116: $2117-2124$

19 Coffee B, Zhang F, Ceman S et al: Histone modifications depict an aberrantly heterochromatinized FMR1 gene in fragile X syndrome. Am J Hum Genet 2002; 71: 923-932.

20 Pomponi MG, Neri G: Butyrate and acetyl-carnitine inhibit the cytogenetic expression of the fragile $\mathrm{X}$ in vitro. Am J Med Genet 1994; 51: 447-450.

21 Tassone F, Hagerman RJ, Taylor AK et al: Elevated levels of FMR1 mRNA in carrier males: a new mechanism of involvement in the fragile-X syndrome. Am J Hum Genet 2000; 66: 6-15.

22 Litt MD, Simpson M, Gaszner M et al: Correlation between histone lysine methylation and developmental changes at the chicken beta-globin locus. Science 2001; 293: 2453-2455.

23 Torrioli MG, Vernacotola S, Mariotti P et al: Double-blind, placebo-controlled study of L-acetylcarnitine for the treatment of hyperactive behavior in fragile X syndrome. Am J Med Genet 1999; 87: 366-368.

24 Pascale E, Battiloro E, Reale GC et al: Modulation of methylation in the FMR1 promoter region after long term treatment with L-carnitine and acetyl-L-carnitine. J Med Genet 2003; 40: e76.

25 Cheutin T, McNairn AJ, Jenuwein T et al: Maintenance of stable heterochromatin domains by dynamic HP1 binding. Science 2003; 299: 721-725.

26 Kouzarides T: Histone methylation in transcriptional control. Curr Opin Genet Dev 2002; 12: 198-209.

27 Pietrobono R, Tabolacci E, Zalfa F et al: Molecular dissection of the events leading to inactivation of the FMR1 gene. Hum Mol Genet 2005; 14: 267-277. 